\title{
DOPPLER IMAGES OF SPOTTED LATE-TYPE STARS
}

\author{
Steven S. Vogt \\ Lick Observatory, Board of Studies in Astronomy and Astrophysics \\ University of California, Santa Cruz, CA 95064
}

\begin{abstract}
Doppler imaging is a technique for deriving resolved images of rapidly rotating stars from a detailed analysis of very high signal-to-noise high resolution spectral line profiles. An improved version of this technique is presented, which now uses principles of maximum entropy image reconstruction to invert the line profile information. The effects that noise, finite resolution, and uncertainties in the assumed stellar physics have on the resultant images were explored through various test simulations. The technique is found to be efficient, accurate, and robust at deriving images of certain classes of stars from realistic quality data. Doppler images are presented of two spotted late-type stars, the RS CVn-type star HR 1099, and the FK Com-type star HD 199178. Both stars show surprisingly similar spot distributions. In each case, there is a single large cool spot straddling the pole, and a number of small cool spots at low latitudes. We expect that the small low latitude spots on each star will migrate poleward to join the polar spot, and suspect that the observed long-lived polar spots are the result of the poleward migration and merging of many active region complexes. If true, the poleward migration of starspots suggests that magnetic activity on very rapidly rotating stars is qualitatively different than that seen on our Sun. We suggest that the observed rotational trigger velocity for the appearance of large spots on late-type stars marks the transition from solar-type boundary layer dynamos to distributed dynamos, which occur only in more rapidly rotating stars. The sizes, locations, and migrations of the spots, however, may be more a result of the convective flow patterns than of any dynamo action, since the spots are quite long-lived.
\end{abstract}

\section{1: INTRODUCTION}

We have developed a technique for deriving resolved images of certain types of rapidly rotating stars. Collaborating with me in this task are my graduate students G. Donald Penrod and Artie Hatzes. We call the method 'Doppler imaging' since it exploits the correspondence between wavelength position across a rotationally Doppler-broadened spectral line and spatial position across the stellar disk to derive a 2-d image of the star. Cool spots and/or abundance peculiarities 
on a rotating star produce distortions in the star's spectral line profiles. If the line profiles are dominated by rotational broadening, a high degree of correlation exists between the position of a given distortion in a line and the longitude on the star of the feature which produced it. A high resolution spectrum of the line is thus, to first order, a 1-d image of the star in longitude, but completely blurred in latitude. As the star is observed from other aspects (other rotation phases) other 1-d images are obtained. Then, if the inclination, rotation period, and other basic physical parameters of the star are known, the set of 1-d images can be combined into a 2-d image of the star in much the same way as the technique of CAT scanning in medical imagery.

The term 'Doppler imaging' has apparently come to mean different things to different people, and is now being used by a number of different researchers to describe various procedures by which spatial information on a star is derived from Doppler signatures in spectral lines. However, there is an important distinction to be drawn. In all other cases of which I am aware, these methods involve just mathematically parameterizing the expected image with some simple function and then solving for the appropriate image parameters by model fitting (for example, fitting spherical harmonic models to non-radially oscillating stars to describe the surface velocity fields, or fitting spotted star line profiles with simple circular spot models). Such approaches, while often informative, are not actually 'imaging' methods since they make highly artificial $a$ priori assumptions about the appearance of the expected image. The technique we refer to as Doppler imaging makes no such a priori assumptions about the image. The resultant image can be arbitrarily complex, and its information content is determined by the size and quality of the data set.

The problem of deriving maps of abundance peculiarities on Ap stars has been around for many years, and a variety of solutions to the problem have been developed (Deutsch 1958, 1970; Pyper 1969; Falk and Wehlau 1974; Megessier 1975). However, all of these approaches involved parameterizing the surface distribution as a truncated series expansion of spherical harmonics or the line profile data as a truncated series expansion of residual intensities. To keep the problem tractable, only a few terms were carried in the expansions. Thus, while the solutions were often mathematically elegant, they were highly constrained artificially and thus were not true 'imaging' methods. More recently, Khokhlova and co-workers (Khokhlova 1975; Khokhlova and Ryabchikova 1975; Goncharsky et. al. 1982; Khokhlova 1985; Khokhlova, Rice and Wehlau 1986) used an approach which could more suitably be called 'imaging'. Their method involved representing observed spectral lines by an integral equation of local equivalent width integrated over the stellar disk, and used a regularizing algorithm to solve for the local equivalent width map. However, their maps generally failed to recover any latitude information about surface features, and their solutions were always strongly biased to the sub-observer latitude on the star.

We have been studying a number of rapidly rotating spotted late-type stars which show time variable distortions in their line profiles. The distortions are attributable to dark spots being carried into and out of view by stellar rotation, as explained by Vogt and Penrod (1983a, 1983b). It was clear from the quite complex nature of these distortions that a much higher degree of spatial information was present in the data than any of the previous line profile inversion methods could cope with. Also, to extract useful astrophysics from images of the spotted stars, we needed a method which gave accurate and reliable latitude in- 
formation, so that spot locations and migration in latitude could eventually be used to measure differential rotation and spot migration rates. Thus we set out to develop a true 'imaging' solution to the line profile inversion problem, one that made no a priori assumptions about the nature of the features to be imaged, and which did not constrain the image solution artificially. The technique we developed incorporates principles of maximum entropy image reconstruction and is proving to be quite robust, efficient, and accurate at deriving resolved images of certain types of rapidly rotating stars.

We are presently using Doppler imaging to study cool starspots on RS CVn and FK Com stars, and surface abundance distributions on Ap stars. The Ap star imaging is being done principally by Mr. Artie Hatzes as part of his Ph.D. dissertation research and will be presented elsewhere in this meeting. Here, I will try to give a brief review of the technique of Doppler imaging and what it has revealed about the nature of giant starspots on certain late-type stars. A more detailed account of the method is currently in press and will appear later this year (Vogt, Penrod, and Hatzes 1987 - here after referred to as VPH). Previous discussions leading up to the present version of the technique can be found in Vogt and Penrod (1983a, 1983b). I was also asked to present a review of Doppler imaging last week at the 27th Liege International Astrophysical Colloquium, so parts of the present discussion of the method will be largely a duplication of that review.

\section{WHAT KIND OF STAR CAN BE DOPPLER IMAGED?}

In order for a star to be suitable for Doppler imaging, it must be rotating fast enough that its line profiles are dominated by rotational broadening. The number of resolution elements across the disk is roughly $2(v \sin i) / W$ where $v$ sin $i$ is the star's projected equatorial rotation velocity and $W$ is the full width at half depth of the star's average specific intensity profile (i.e. that which would be obtained from a flux spectrum of the star in the absence of stellar rotation). For active late-type dwarfs and subgiants, $W$ is typically about $10 \mathrm{~km} \mathrm{~s}^{-1}$, so a minimum $v \sin i$ of 20 to $30 \mathrm{~km} \mathrm{~s}^{-1}$ is needed to get any useful amount of resolution. The larger the $v \sin i$, in principle, the better the resolution. However, since line equivalent width is conserved, the lines become shallower as the $v$ sin $i$ is increased, and the distortions, which are always some fraction of the line depth, become weaker. Above 80 to $100 \mathrm{~km} \mathrm{~s}^{-1}$, the distortions become very difficult to detect and disentangle from line blends and weak terrestrial features. So projected velocities of 40 to $60 \mathrm{~km} \mathrm{~s}^{-1}$ are about ideal for late-type stars. For hotter stars with less line blending and/or stronger lines, larger $v$ sin i's are usable.

In order to be able to assemble line profile data from many different rotational aspects of the star, the star's rotation period must be known. Fortunately, this is almost always well determined by broadband photometry. However, we have encountered cases where the published photometric periods were slightly incorrect, and the Doppler imaging technique could not converge on a solution. In such cases, the imaging technique itself was used to provide a much more accurate period as part of the solution.

The stellar inclination must also be at an intermediate value. Poleon orientations have no line-of-sight components of surface velocity and thus no Doppler information. Equator-on orientations suffer a north/south hemispheric 
ambiguity and yield Doppler images with mirror-like symmetry about the equator. However, quite a wide range of inclinations are possible, and the method is fortunately quite insensitive to errors in the assumed inclination. In practice, we have found inclinations from $20^{\circ}$ to $70^{\circ}$ to be feasible.

The basic physical properties of the stellar atmosphere must also be known, but, fortunately, not to any high degree of precision. We have found that an LTE-level of approach to the line synthesis is quite adequate. We treat the limb angle dependence of line strengths, shapes, and continuum brightness explicitly in the method.

\section{DATA REQUIREMENTS}

Doppler imaging requires extremely high quality spectral data. The data consist of a set of high resolution, high signal-to-noise spectral line flux profiles obtained at different rotation phases. For the spotted late-type stars, we use either the $\mathrm{Fe}$ I line at $6431 \AA$, or the Ca I line at $6439 \AA$. Both are relatively unblended and reasonably clear of terrestrial absorption features. For Ap stars, we use either the Cr II line at $4824 \AA$ or the Si II line at $6347 \AA$. In general, we only use a single atomic species of spectral line for each image, but the method can be set up to use many different lines simultaneously. While this complicates the process, it could provide additional constraints on the image. To take full advantage of the resolution available at the star, the instrumental profile must be no larger than the star's intrinsic line width. A resolving power of at least 40,000 is thus preferred for the spotted late-type stars, though some useful information may still be obtainable at lower resolutions.

The line profile distortions are typically only $1 \%$ or less of the continuum, so quite high signal-to-noise data are required to achieve reliable images. We aim for a $S / N \geq 400$ per $0.13 \AA$ resolution element, though our simulations (VPH) indicate that useful information is still obtainable down to $\mathrm{S} / \mathrm{N}$ of about 150-200 per resolution element. To obtain complete images of the visible portion of the star, line profiles must be well distributed over rotational phase space. A minimum of 8 to 10 profiles distributed evenly over phase space is required for a good image. Images derived from uneven phase coverage may still be useful, but will lose resolution and become less unique.

The line profile data are obtained with the coudé spectrometer of the Lick Observatory Shane 3-m telescope. We use a Texas Instruments $800 \times 8003$ phase CCD (Robinson and Osborne 1986), at the focus of the 80" coudé camera. With the 9.5" collimator and Grating VII in second order at $6435 \AA$, this set-up results in a dispersion of $38 \mathrm{~m} \AA$ per CCD pixel, and spectral coverage of $30 \AA$. We also use a Bowen-Walraven image slicer which not only increases throughput at the slit, but also 'cools' the image down in one dimension at the focal plane by spreading the light perpendicular to dispersion over many rows of the CCD. Thus, less dynamic range is required from the CCD and high signal-to-noise exposures are easier to obtain. The slicer accepts light from a $3^{\prime \prime}$ diameter entrance aperture, and slices it down to a projected slit of 3.4 pixels at the focal plane or $0.129 \AA$. The resultant resolution is 50,000 at $6435 \AA$.

The line profiles must be carefully reduced before attempting any imaging. Besides the usual flat fielding and zero point corrections which are necessary with most array detectors, the data must be thoroughly cleaned of telluric ab- 
sorption features and, if the star is in a binary system, the spectra of the companion star(s) must also be carefully removed. Fortunately, with modern digital data and computers, this is relatively straightforward, but must be done with great care as the Doppler imaging signatures are quite small.

\section{INVERTING THE SPECTRAL LINE FLUX PROFILES}

Each line profile is basically a 1-d marginal brightness distribution or projected image of the stellar disk. The task of 'inverting' the line flux profiles to derive the brightness distribution across the stellar surface is thus basically a deprojection problem, and is best handled in matrix form on a computer. We have then an image space and a data space, with some matrix which transforms between them in one direction.

\subsection{Set Up The Data Vector}

Once each profile is fully reduced as described above, the set of all profiles are phased using the known rotation period and assembled end-to-end with just enough separation between to reach the continuum between profiles. Figure 1 shows a complete data set for the star HR 1099 from the Fall 1985 observing season, using the Ca I $6439 \AA$ line. The crosses indicate the actual data, and the solid line represents our imaging software's final fit to these data (to be discussed later). Since the spots change significantly on a time scale of 4 to 5 months on these stars, all the line profiles were obtained in less than a 3 to 4 month interval. This set of data points defines our data vector $\mathbf{D}_{k}$, and typically contains up to 1000 points.

\subsection{Set Up The Image Vector}

We create an image space by subdividing the star up into a grid of surface brightnesses, and expressing these surface brightness values as a simple 1-d image vector $\mathbf{I}_{j}$. Figure 2 shows how the star is sectioned. We use a grid of zones whose edges lie along lines of longitude and latitude, and vary the number of zones at each latitude such that the true area of each zone is roughly constant over the star. Thus, zones at high latitude span a larger range in longitude than zones at low latitudes. The minimum number of zones required is constrained by the need to adequately resolve features on the star. The maximum number allowed is constrained by the need to keep the problem of manageable size for our VAX 11/780 computer. In practice, zone sizes near the equator of about $5^{\circ}$ by $5^{\circ}$ are sufficient to provide adequate sampling of the disk. So the number of zones used is typically 1200 to 2500 . Here, the surface is divided up into 40 latitude bands. At this inclination of $40^{\circ}$, a total of 2310 zones are required to cover the visible portion of the star. Each zone is assigned a corresponding surface brightness. The set of all such surface brightnesses comprises our one-dimensional image vector.

\subsection{Create The Response Function Matrix}

We then set up a response function matrix $\mathbf{R}$ that transforms from image space to data space. The creation of this matrix is essentially the same procedure one goes through to synthesize a line flux profile from a star of known atmospheric and geometrical parameters. It involves working the problem forward using an LTE model of the stellar atmosphere and knowledge of the star's inclination, and 

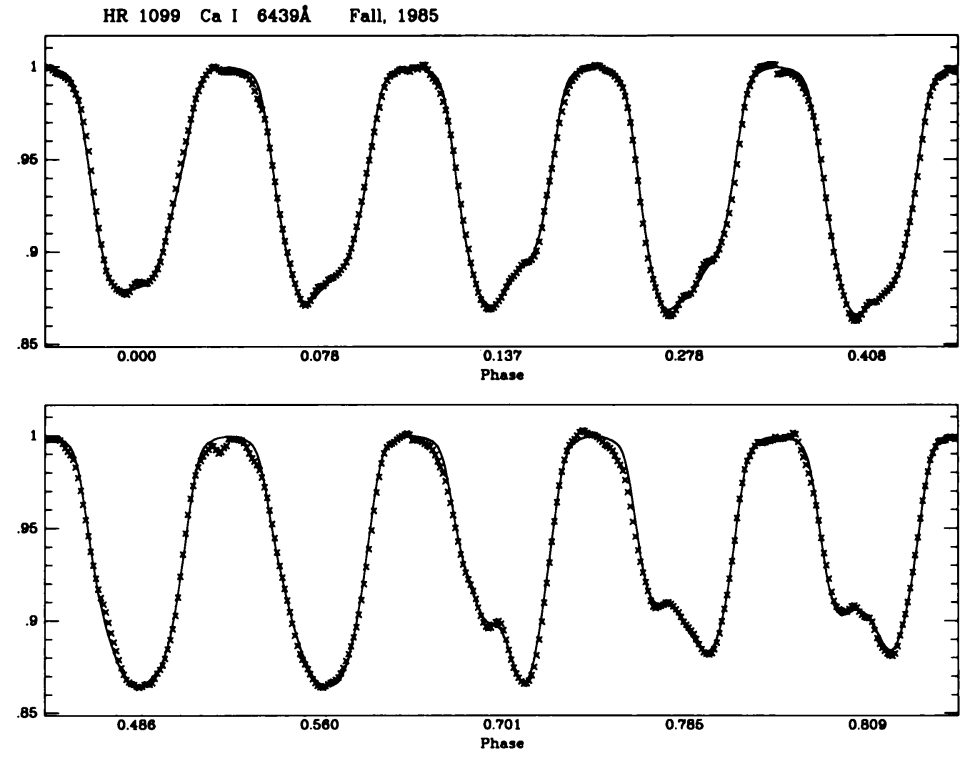

Figure 1. Data vector for HR 1099 in 1985.

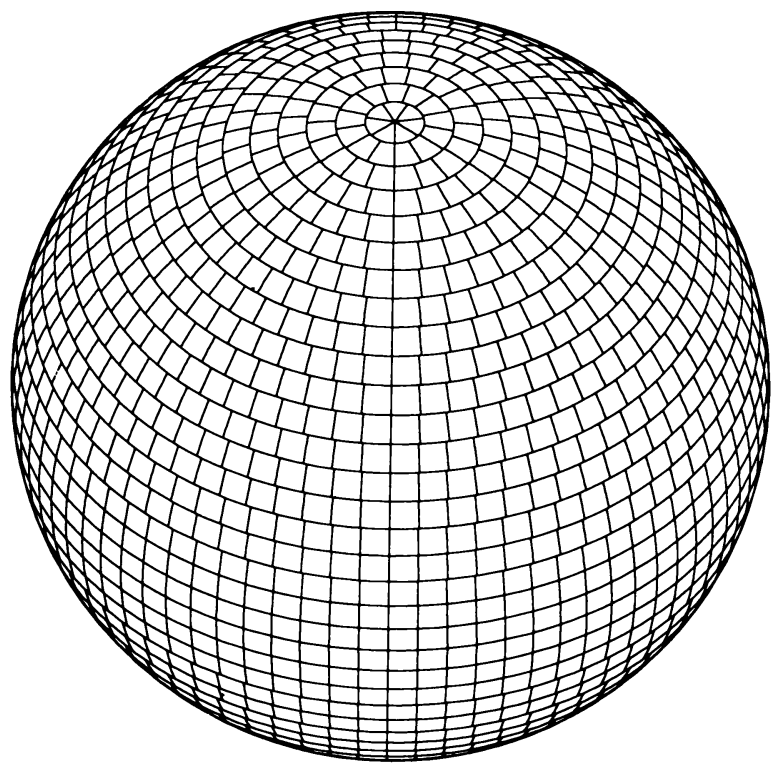

Figure 2. Zonal division of the stellar surface. 
ically different in the 'spots' than in the photosphere, and in which both spots and photosphere make a significant contribution to the observed line, then some more elaborate prescription may need to be used. For our case, however, since the spot does not make a significant contribution to the observed flux, we set $\mathrm{dI}_{\lambda} / \mathrm{dI}_{c}$ equal to $I_{\lambda} / I_{c}$ (where $I_{c}$ is the continuum surface brightness and the $I_{\lambda}$ 's the specific intensities across the spectral line), which much simplifies the computing.

The radial velocity of the zone due to rotation is then calculated. The appropriate values of $\mathrm{dI}_{\lambda} / \mathrm{dI}_{c}$ across the line profile are then multiplied by the effective area of the zone, and shifted in wavelength according to the correct radial velocity. These values then become the response matrix elements for the data pixels obtained at that phase for that particular zone on the star. They tell how changing the surface brightness of that zone will affect the rotationally broadened line profile of the star at that particular phase. The entire transformation matrix, $\mathbf{R}$, consists of all these values, for each zone on the star and for each data phase.

\subsection{Inverting the Response Function Matrix}

Once the problem is set up in the form $\mathbf{I} \mathbf{R}=\mathbf{D}$, one could ideally just solve directly for the image vector by inverting $\mathbf{R}$ and solving for $\mathbf{I}$. Thus, $\mathbf{I}=\mathbf{D} \mathbf{R}^{-1}$. There are, however, a number of problems with this. $\mathbf{R}$ is not generally square and thus any given solution to the matrix equation is not unique. Furthermore, its rows are not independent. Effects such as projection along chords of constant radial velocity, finite width of the star's intrinsic line profiles and of the instrumental profile, incomplete phase coverage, and phase smearing during exposures all contribute to yield rows which are not independent. Also, even if $\mathbf{R}$ were well enough conditioned to be directly invertable, the data always contain some noise. Any noise features in the data would thus tend to produce spurious features in the image.

What is needed is a way to iteratively transform between image and data space, searching among all possible image vectors until one is found which fits all the observed data to within the known noise level of those data. The number of possible image vectors consistent with the data set can be large, and one must set some criterion for selecting the 'best' solution. One likely criterion is to choose the simplest or smoothest image, i.e. the one with the least amount of information, which is still consistent with all the data to within the known noise level of the data.

This type of problem is commonly referred to as digital image reconstruction, and is often encountered in radio astronomy, and medical imaging. A number of techniques have been developed for reconstructing an image from an incomplete and noisy data set. Perhaps the most widely used and powerful of these is the maximum entropy method (Skilling and Bryan 1984 and references therein), realized in software as MEMSYS, a computer program developed by Drs. J. Skilling and S. F. Gull. Of the various known digital image reconstruction techniques, maximum entropy has been shown to be the optimal technique of image reconstruction, and the only consistent regularization technique for images (Shore and Johnson 1980, 1983; Gull and Skilling 1983). In addition, the MEMSYS implementation of image reconstruction has proven to be extremely robust and efficient, and has seen wide application in astronomy, medical imaging, forensic imaging, and elsewhere. Subsequently, the MEMSYS software was kindly made available to us by Dr. Gull through Dr. R. Padman of the University of California at Berkeley Radio Astronomy Laboratory. 
rotational velocity to set up a system of some 1000 equations of approximately 2000 terms each which relates all 2000 values of surface brightness on the star to all 1000 data points produced by the spectrometer. Thus,

$$
\begin{aligned}
& I_{1} R_{11}+I_{2} R_{21}+\ldots \ldots \ldots \ldots \ldots+I_{n} R_{n 1}=D_{1} \\
& I_{1} R_{12}+I_{2} R_{22}+\ldots \ldots \ldots \ldots \ldots+I_{n} R_{n 2}=D_{2} \\
& I_{1} R_{1 m}+\dot{I_{2}} R_{2 m}+\ldots \ldots \ldots+I_{n} \dot{R}_{n m}=D_{m}
\end{aligned}
$$

or $\mathbf{I} \mathbf{R}=\mathbf{D}$.

The matrix $\mathbf{R}$ represents the response of our measurement apparatus, and is analogous to the point spread function of an imaging system. In our case, the 'imaging' system is a high resolution spectrometer which measures stellar flux profiles and is situated at some inclination with respect to the star's rotation axis, working at a particular wavelength, and observing at a specific set of rotation phases. Each $\mathrm{R}_{j k}=\frac{\partial D_{k}}{\partial I_{j}}$ and represents the response of data pixel $\mathrm{k}$ to changes in the surface brightness of image zone $\mathrm{j}$. The $\mathbf{R}$ matrix is of course $n$ by $m$ in size, so for our typical cases, with $n \approx 2000$ and $m \approx 1000$, it requires (in byte form) about 2 Megabytes of memory. Each set of line profiles of a given star from a given epoch requires its own unique $\mathbf{R}$, since, although the basic stellar parameters do not change, the exact phases, choice of lines, and instrumental resolution generally vary from one season to the next.

The continuum intensity and intrinsic shape of the spectral line profile from any localized spot on the star are both functions of limb angle. We first calculate the local specific intensity profiles for $30 \mathrm{limb}$ angles using the ATLAS 5 subroutines from Kurucz (1979) and model atmospheres from a variety of sources. These profiles are then convolved with a typical radial-tangential macroturbulent velocity function and with the instrumental profile. At different phases, a typical zone on the stellar surface will have different (1) projected area, (2) limb angle, and (3) radial velocity (due to stellar rotation). Since we want to know how changing the surface brightness in a given zone will affect the observed rotationally-broadened profile at each phase, each of these quantities must be calculated for each zone at each phase. At each phase, the program first calculates the $x-y$ position on the stellar disk corresponding to each latitude-longitude zone. At many phases (if the zone is not circumpolar) a given zone will be on the back side of the star and will not be visible. The effective areas of these zones are set equal to zero. For the zones that are visible, the limb angle is calculated. This then tells the code both which set of specific intensities to use and the projected effective area of that zone at that phase.

For simplicity, we assume that the shape and strength of the line profile at each limb angle is the same in the 'spots' as in the 'photosphere'. In our case, where we expect the spots to be virtually black anyway, this assumption is not critical, and much simplifies the computational process. It should be noted, however, that if the line equivalent width is different in the spot than it is in the photosphere, what we are really mapping is a combination of surface brightness and line strength. In cases in which the shapes of the spectral lines are dramat- 
Maximum entropy image reconstruction involves finding the image with the greatest configuration entropy:

$$
S=-\sum_{j} p_{j} \log \left(p_{j}\right)
$$

where $p_{j}$ is a normalized positive dimensionless image quantity (surface brightness in our case) of the $j$ th image pixel, and the sum is taken over all $n$ image pixels. The maximum entropy image is the one with the least amount of spatial information, and is thus the smoothest or simplest image. In our case, the 'smoothest' image means the one with the least contrast between spots and stellar photosphere. An immaculate star, with no spots, will thus be the image with the maximum entropy but it will not fit the observed line profiles of a spotted star. MEMSYS uses a multi-dimensional search algorithm to find its maximum entropy image subject to the additional constraint that the image produces data which fit all the real data to within some level $\chi_{0}^{2}$ where $\chi^{2}$ is a measure of the misfit:

$$
\chi^{2}=\sum_{k}\left(g_{k}-d_{k}\right)^{2} / \sigma_{k}^{2}
$$

Here, $g_{k}$ is the data set produced by the image solution and $d_{k}$ is the actual data, whose uncertainty at any point is $\sigma_{k}$.

MEMSYS accomplishes the constrained maximization by the method of Lagrange multipliers, using $\mathbf{R}$ and its transpose $\mathbf{R}^{\prime}$ to iterate back and forth between image and data space, seeking to maximize the functional:

$$
Q=S-\lambda \chi^{2}
$$

where $\lambda$ is the Lagrange multiplier. It accomplishes its search very efficiently through the use of conjugate gradient techniques and a 6-dimensional subspace of search directions, rather than doing simple line searches. For linear image reconstruction problems, surfaces of constant $\chi^{2}$ are convex ellipsoids in N-dimensional image space. Since the entropy surfaces are strictly convex, the maximum entropy reconstruction is thus unique (Skilling and Bryan 1984).

In practice, the $S / N$ in our actual line profile data is so high that the image solution is well defined by the $\chi^{2}$ term only. That is, by the time the iteration has succeeded in fitting the data to the required level of accuracy, further improvements in maximizing the entropy have no appreciable effect on the image. Thus our Doppler images are well constrained by the data set alone and do not rely heavily on entropy considerations. To the level allowed by the data's inherent noise, they are thus unique.

Of course, the star does not care about maximum entropy and there may well be finer structure present in the true image, but it will never be uniquely determinable from the data set. The real beauty about the maximum entropy image and the reason it is particularly informative is that, because it does have minimum configurational information, one is assured that any structure present in the image is actually required by the data. Thus, the signal-to-noise, 
and quantity of data ultimately determines the level of unique detail allowed in the reconstructed image, as it must.

\section{A TEST OF THE METHOD}

We have tested the Doppler imaging software extensively by giving it synthesized spectral line flux data from test images of stars. The reader is referred to VPH for a more complete discussion of these tests. The following test case illustrates how well the method works in the best of all possible worlds using an artificial image of an RS CVn star. The $v$ sin $i$ of the artificial star is $40 \mathrm{~km} \mathrm{~s}^{-1}$ and its inclination is $40^{\circ}$, values typical of our real program objects. The spectral type of the artificial star was taken to be $\mathrm{K} 1 \mathrm{IV}\left(\mathrm{T}_{\text {eff }}=4750 \mathrm{~K}\right)$, and the spectral line used to generate the synthetic data set was Fe I $\lambda 6431 \AA$.

The test image chosen was a rather complicated image consisting of the letters V-O-G-T written in dark spots around our imaginary star. The test image of the 'Vogtstar' is shown in Figure 3 for eight equidistant rotation phases. The phantom star at lower right illustrates the location of the rotational pole and inclination. This image was then used to generate a data set of 16 line profiles of the Fe I $\lambda 6431 \AA$ line at equidistant phases. The synthetic data were generated using an explicit disk integration scheme, using a totally different zone geometry than that used in the reconstruction software, and with the true spectral line shape and strength appropriate for the dark spots explicitly included in the calculations. In this case, however, we made two assumptions intended to maximize the information in the line profiles. First, we assumed that the spectral resolution used was infinite, and second, we assumed that the spots were extremely cool, with $\mathrm{T}_{e} \simeq 2700 \mathrm{~K}$. Both effects tend to make the line profile variations rather more dramatic, and thus give the code more information to work with.

The 16 profiles were assembled end-to-end into a 512 point data vector, and the Doppler imaging software was then given the appropriate physical parameters for the star (inclination, rotation velocity, and specific intensity profiles as a function of limb angle), and asked to derive an image of the star using only these synthetic line profiles. The input line profiles are shown in Figure 4 (crosses), and the fit to those profiles after 30 iterations of the inversion software is shown by the solid line. We let the program proceed until the profiles were fit to a very high level of accuracy.

The image derived by the inversion process, also after 30 iterations, is shown in Figure 5 at the same eight phases as the input image, and gives a good view of the level of the success of the inversion procedure. Though there is some loss of resolution, as expected, the test image has been recovered very well, certainly well enough to recognize all the letters, and to see a distinct difference between the ' $\mathrm{O}$ ' and the ' $\mathrm{G}$ ' letters. The effective resolution is approximately $12^{\circ}$. The recovered image is free from any systematic limb brightening or limb darkening effects, and also shows no systematic latitudinal brightening or darkening effects. Clearly, in this best of all worlds, the inversion software works very well. The main features of the solution are very well constrained by the data, and do not rely heavily on entropy assumptions. 


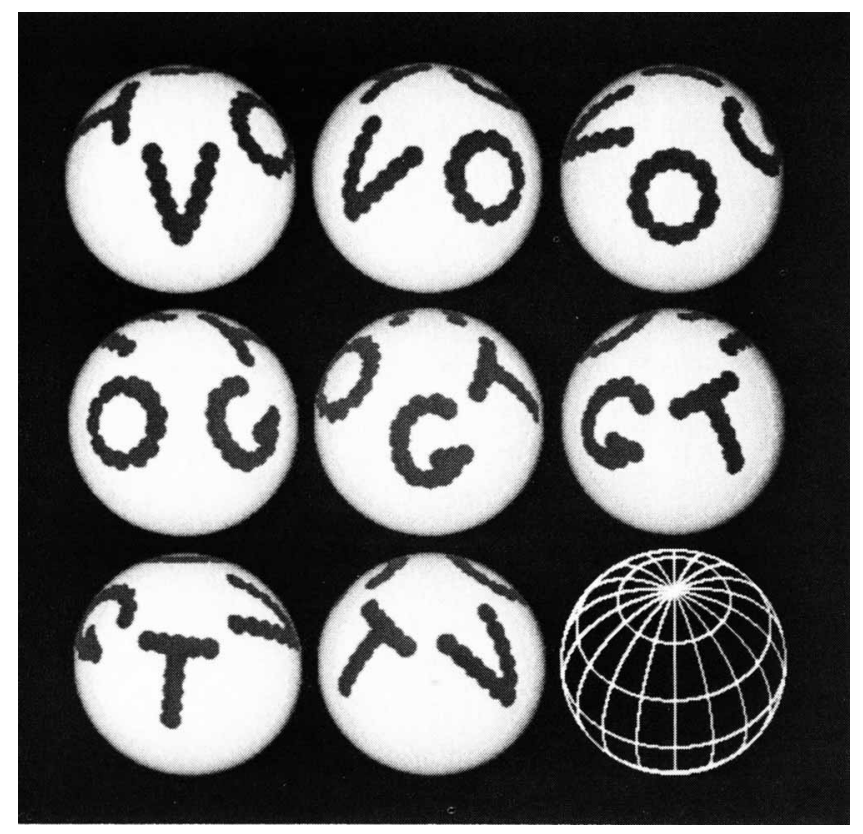

Figure 3. Test input image of the 'Vogtstar'.

\section{DOPPLER IMAGES OF SPOTTED STARS}

We are currently observing a number of RS CVn, FK Com, and Ap stars with Doppler imaging. For the RS CVn and FK Com stars, we are attempting to monitor the appearance and evolution of large starspots on a select sample of stars from year to year for information on the nature of magnetic dynamo processes within these stars. For the Ap stars, we are studying the nature of abundance patterns of various ions across the stellar surface. Magnetically confined diffusion of certain atomic species seems to be at work in the atmospheres of the peculiar A stars, and our Doppler imaging studies are aimed at providing observational confirmation of the diffusion theories as well as yielding information about the magnetic fields of stars which rotate too rapidly for conventional Zeeman analysis techniques.

Figure 6 shows a Doppler image of HR 1099 in 1981. This star is one of the brightest members of the RS CVn class of spotted late-type binaries and is very well-suited to the Doppler imaging method. The orbital period was used for the phasing of this and all subsequent HR 1099 images. The 1981 spot configuration consisted of a spot situated almost directly at the pole, with an attached protuberance descending to lower latitudes. Another small isolated spot lies below, at the equator. It is curious that the longitude of the protuberance on the polar spot coincides precisely with the extrapolated longitude of the migrating 

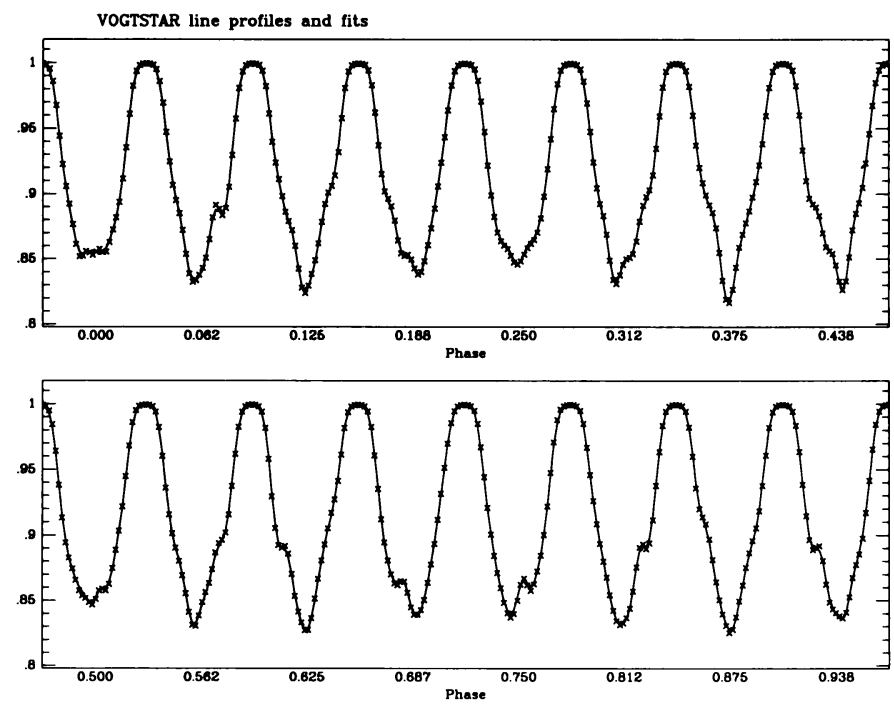

Figure 4. Synthetic line profiles for 'Vogtstar'.

large active region complex which emerged near the equator of this star in 1978 . Though it is still too early to draw definitive conclusions from such coincidences until more is known about spot migration patterns, one is tempted to conclude that the equatorial complex of 1978 migrated poleward for 3 years, and, in 1981, was seen merging with the polar spot.

Figure 7 shows HR 1099 in 1984. The polar spot still has an attached protuberance though it's not yet clear if it's the same one as in 1981. There are also a number of low latitude spots, one pair of which bears a striking resemblence to a classical bi-polar sunspot group, with leading and trailing spots. Clearly, the spot distribution has changed dramatically between 1981 and 1984, though the polar spot is basically unchanged and appears to be a long-lived feature. Figure 8 shows HR 1099 in 1985. The polar spot is still basically unchanged, but now has a very large attached protuberance. Since we generally can manage to get only one image per year, it is difficult to know which features are the same and how they have moved from one year to the next. At the rate of one image per year, we may very well be undersampling the changing spot morphology.

We currently have imaging data on HR 1099 from 1981, 1982, 1984, 1985 , and 1986. The 1983 season was lost to bad weather. When all these images are in final form, they will be combined and used to (hopefully) track the various spots from year to year. In this way, we hope to get a direct measure of latitudinal shear (differential rotation) and gain further understanding of cyclic dynamo processes at work within such stars. A very preliminary look at the present set of images on HR 1099 seems to suggest that spots initially form at low latitudes and 


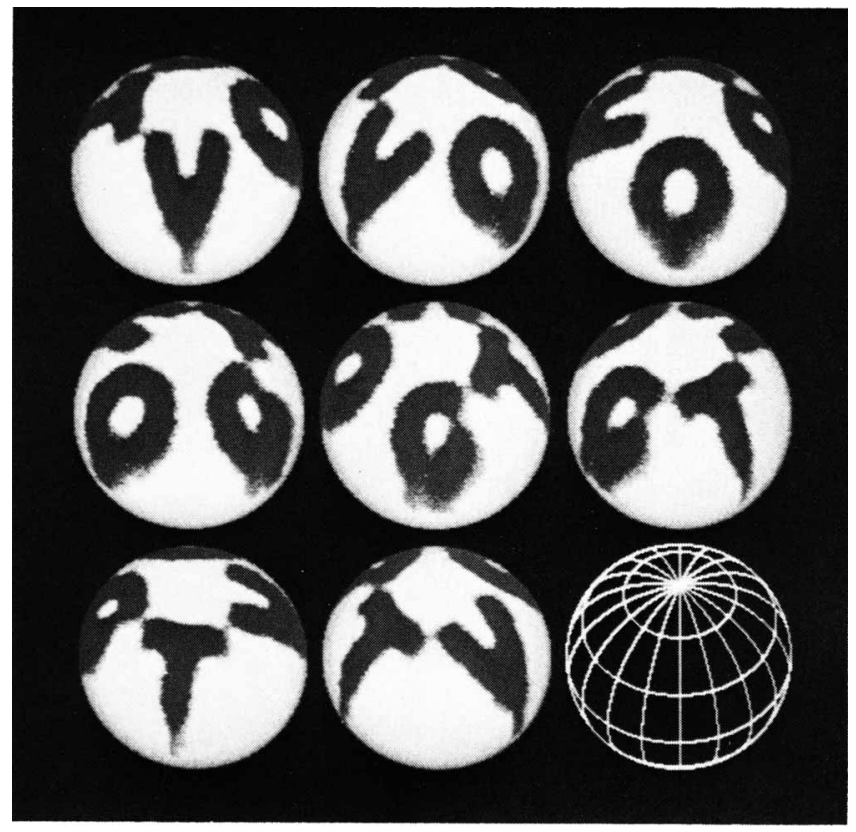

Figure 5. Doppla: image recovery of 'Vogtstar'.

then migrate poleward to join a long-lived polar spot.

HR 1099 is about 120 light years away, and totally unresolvable with existing telescopes. To put things in perspective, the resolution achieved here with Doppler imaging (about $10^{-4}$ arc seconds) is equivalent to resolving Abraham Lincoln's ear on a penny viewed from 3000 miles. Achieving the same resolution with an optical telescope would require a diffraction-limited space-borne telescope of about 1 mile aperture. Providing the photon flux is adequate, the linear resolution of our Doppler images is, of course, independent of stellar distance.

Figure 9 shows a Doppler image of the star HD 199178 obtained in 1985. This star is a rapidly rotating late $G$ giant and a member of the FK Com class. Stars of this class are rapidly rotating late G-K giants. They are apparently single stars with a high degree of surface activity, and may well be recently coalesced binaries. Again, one sees a very large polar spot situated almost directly at the pole, and a single smaller isolated spot at lower latitude. As with HR 1099, the polar spot may be quite long-lived, and is probably the result of the merging of many smaller spots which originally appeared at lower latitudes and migrated poleward to join the polar spot.

\section{DISCUSSION}

These first Doppler images of starspots revealed (to us at least) a striking resem- 


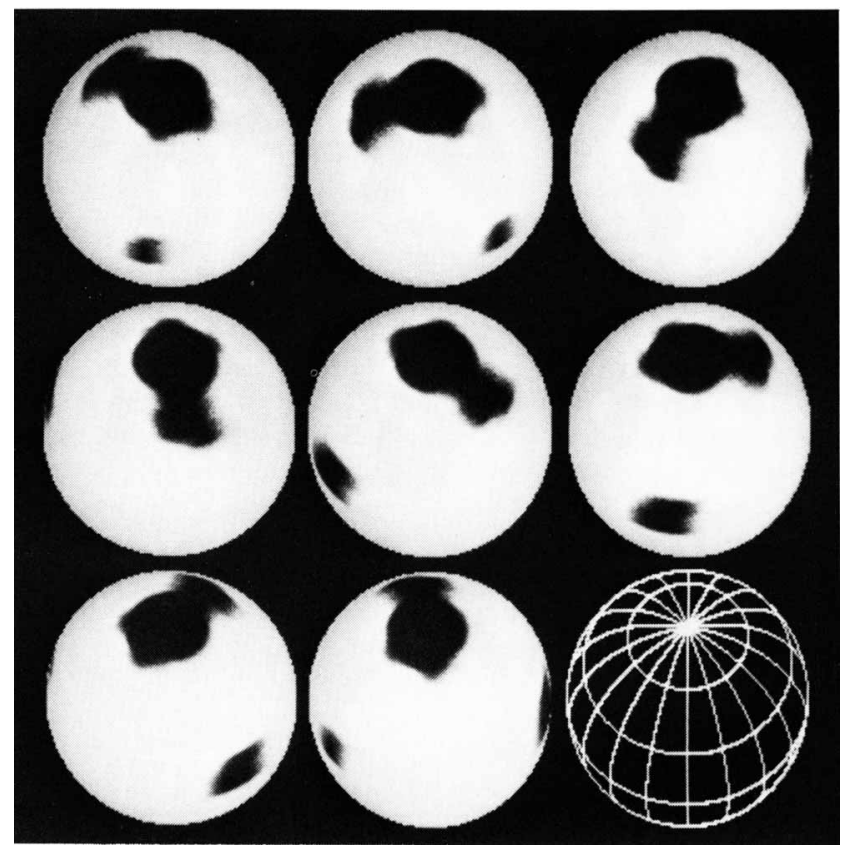

Figure 6. Doppler image of HR 1099 in 1981.

blance to x-ray images of solar coronal holes. In both cases, one sees the geometry of a long-lived polar spot, often with an attached protuberance descending to lower latitudes. Smaller spots (holes) first appear at lower latitudes, and in the solar case, migrate poleward to join the polar spot (hole). We believe (Vogt and Penrod 1983b) that starspots are perhaps more analogous to solar coronal holes than to sunspots. Solar coronal holes are prominently visible only in the $\mathrm{x}$-ray, since their magnetic fields are only 10-20 G, and too weak to have any significant effect on radiation transport in the photosphere. It is only in the corona, where the densities are much lower that these weak fields can significantly affect the plasma motions, leading to lower temperatures and lower densities as a plasma wind flows outward above the hole. Thus, coronal holes are almost inconspicuous photospheric structures, but they dominate the structure of the corona, and appear prominently in x-ray coronal images. However, the magnetic flux inside starspots is probably several orders of magnitude larger than in solar coronal holes, yielding kilogauss fields over a convective cell. Such large fields are strong enough to dominate convective heat transport in the photosphere, and the region above the spot turns cool, leading to spots which are prominent in photospheric light. The blocked energy from such a starspot is readily absorbed by the convective envelope, and reradiated over the entire surface of the star over a many decade time scale. So, starspots are probably like sunspots, in that they are cool because magnetic fields dominate convective motions, but they are like coronal 


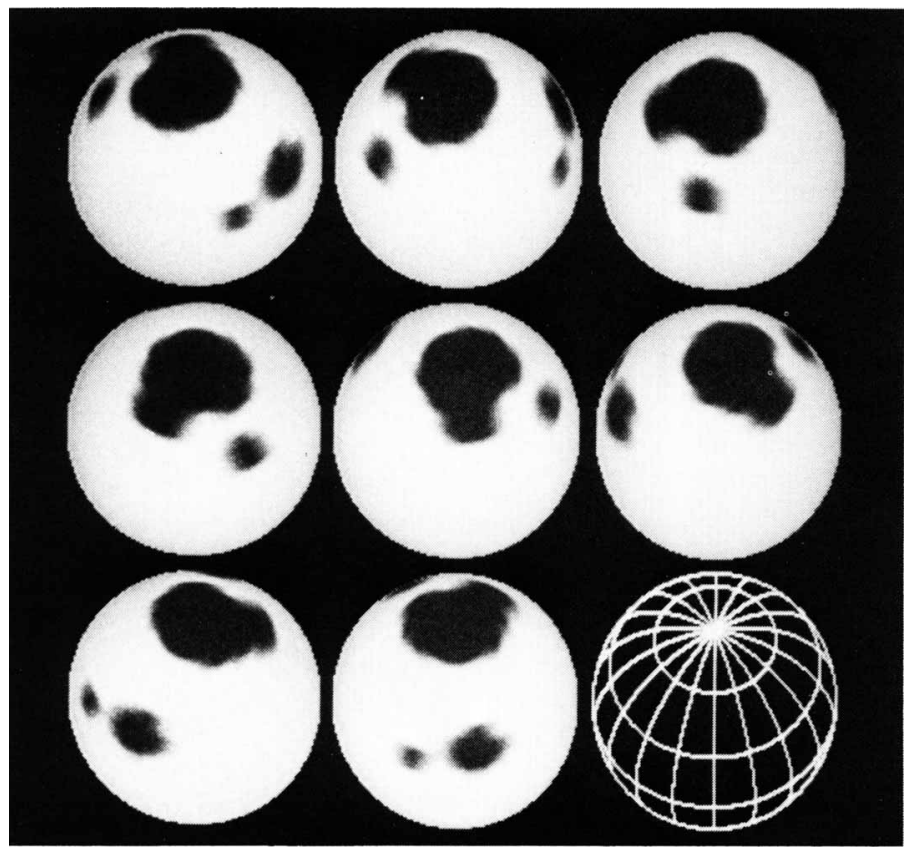

Figure 7. Doppler image of HR 1099 in 1984.

holes in terms of their sizes, and global migration patterns. In particular, they do not appear to show the familiar equatorward migration pattern of sunspots (the butterfly diagram), but rather a poleward migration pattern.

Starspots probably first appear at low latitudes as scaled-up analogs of solar complexes, with many active regions merging to form what we call a starspot. The filling factor of dark umbrae is very much larger than typical of solar complexes, however, because of the much larger emergent magnetic flux. The appearance of new magnetic flux is accompanied by much flaring, enhanced radio and X-ray emission, and chromospheric emission. With time, the activity declines as the fields become organized through magnetic reconnection, annihilation, and diffusion. The resulting unipolar open field regions then migrate to the poles, where they accumulate to form large long-lived polar spots. The size of the polar spot probably varies cyclically, as occurs with polar coronal holes on the Sun.

If the hypothesis of poleward migration of starspots is born out by further analysis of Doppler images, it may be an indication that magnetic activity on these rapidly rotating, deeply convective stars is qualitatively different than that traced by sunspots on our Sun. Models of distributed $\alpha-\omega$ dynamos operating in rapidly rotating convective shells predict poleward migration. Recent models of the solar dynamo, which attempt to explain the observed equatorward migration of sunspot formation zones, suggest that the solar dynamo operates primarily in the layer immediately beneath the convective zone, as a 'boundary 


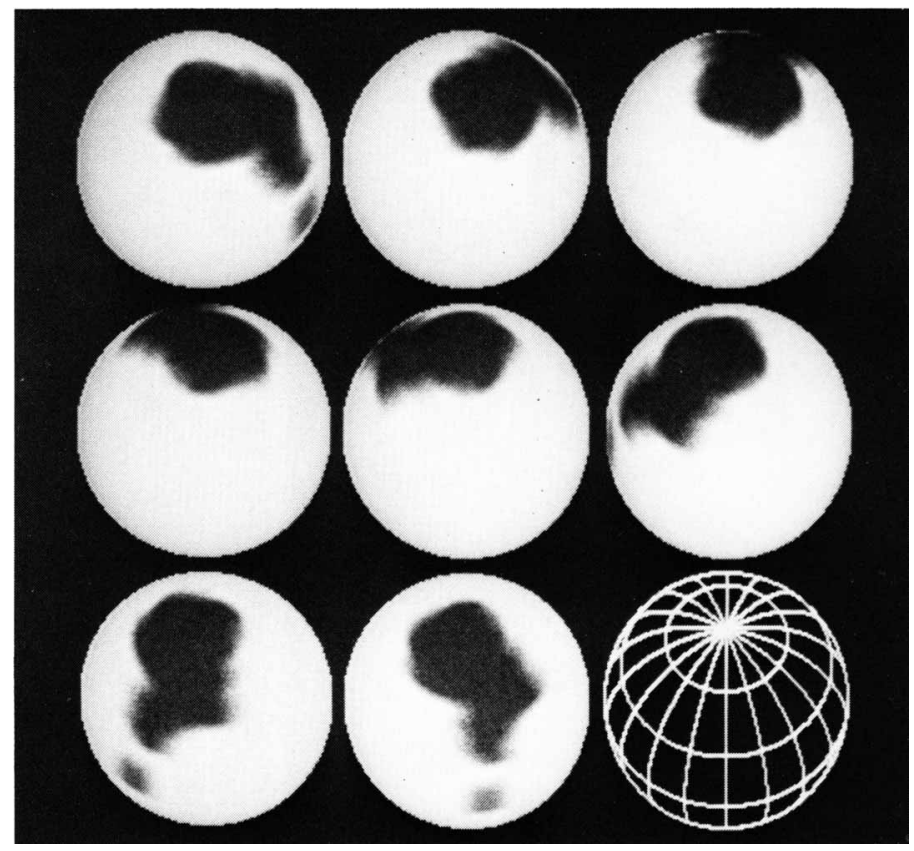

Figure 8. Doppler images of HR 1099 in 1985.

layer' dynamo. It is possible that the solar rotation is not rapid enough to operate a true distributed dynamo, while the more rapidly rotating stars that we observe do exhibit such a dynamo. It is also possible, however, that spot locations and migrations may be determined by the locations of large convective rolls in the convection zone, rather than by anything connected to the dynamo waves, as recent models of the solar activity cycle suggest. In stars with very deep convection zones, such as the ones we observe, it is possible that each hemisphere is dominated by a single convective roll, with magnetic flux rising at the equator and then being transported poleward, to appear as a large polar spot centered over the polar subduction zone.

Hints of periodicity in mean light level variations and in the amplitudes of light curves for a number of spotted stars suggest some evidence for starspot cycles. Periodic variations in the size of the polar spot would yield periodic mean light level variations quite naturally. Figure 10 is an attempt to combine various widely scattered reports of 'cycles' for RS CVn and BY Dra type spotted stars and to merge these with the systematic studies of periodicities of active single $G$ and $\mathrm{K}$ dwarfs done by Vaughan et. al (1981). The figure and data are from Vogt (1983) and are perhaps now a bit out of date, but they do serve to illustrate an interesting point. Among the active late-type stars, the cycle period is essentially constant at around 10-12 years for all stars with rotation periods longer than about 6-7 days, including the Sun. The stars show little evidence of starspots, 


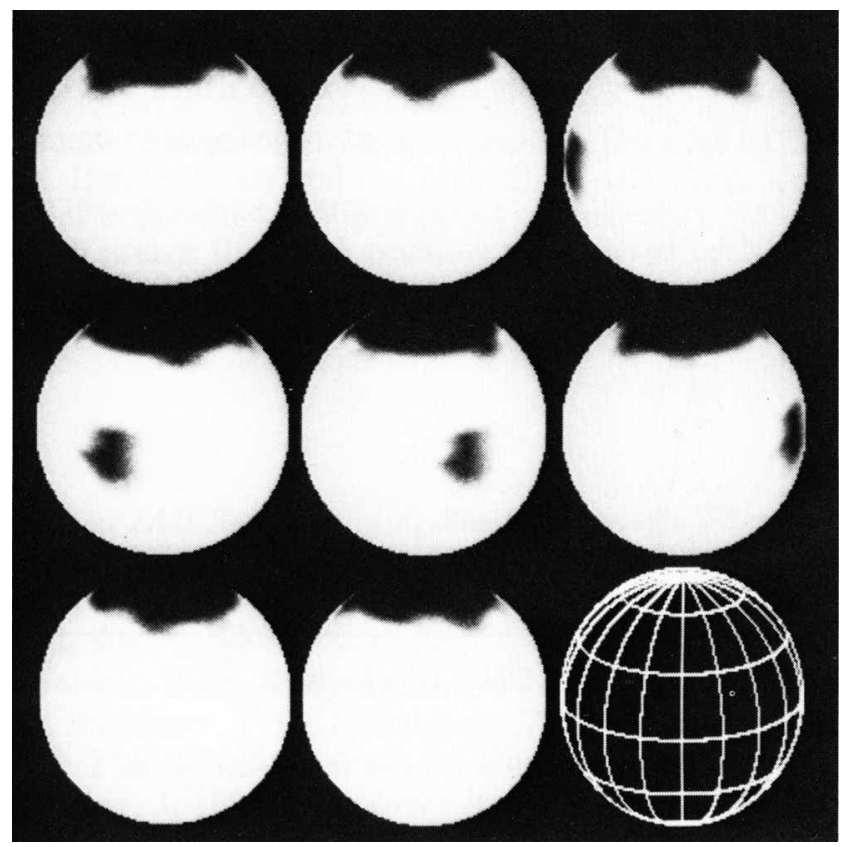

Figure 9. Doppler image of HD 199178 in 1985.

as would be the case for the Sun as viewed from a similar distance, and the cycle period is determined strictly from variations in the level of chromospheric emission. For more rapidly rotating stars, giant starspots appear, and the 'cycle period' increases linearly with decreasing rotation period. There appears to be a threshold rotation rate, corresponding to a 6-7 day rotation period, above which a star forms large starspots. The connection of these 'cycle periods' for the rapidly rotating stars to true dynamo periods is, however, very uncertain.

We suspect that the appearance of large spots on the rapidly rotating late-type stars marks the transition from a feeble solar-type boundary-layer dynamo to a more powerful distributed dynamo which operates throughout a deep rotating convective shell. The sizes, locations, and migrations of the starspots, as well as the 'cycle times', may well, however, be telling us more about the subsurface convective pattern in these deeply convective stars than anything about the true dynamo activity. Doppler imaging studies of cyclic variations in the size of the polar spot, of the frequency of spot formation, and of spot migration patterns should provide a useful tool for unraveling the nature of the very powerful magnetic activity on rapidly rotating stars, and hopefully will lead to a much better understanding of the activity of the most local dynamo, the Sun. 


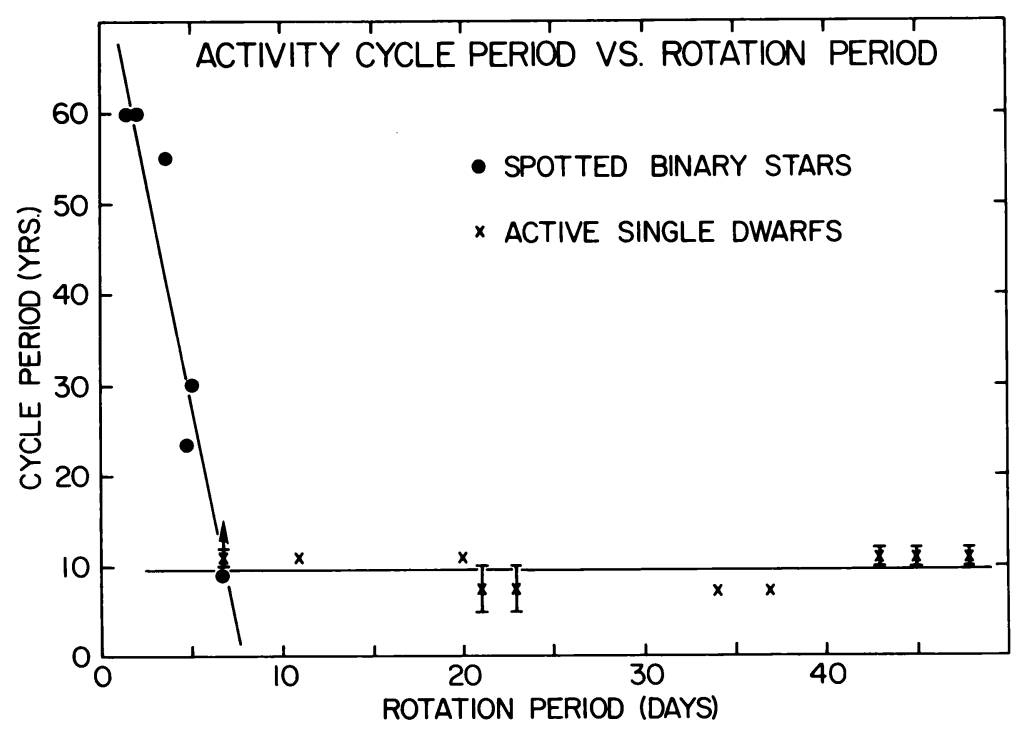

Figure 10. Cycle period vs. rotation period for a sample of active late-type stars.

\section{WHAT'S NEXT FOR DOPPLER IMAGING?}

The technique of Doppler imaging is still in its infancy and further development is underway at Lick. The true line synthesis problem is not a simple linear process with specific intensity line profiles which are independent of temperature or equivalent width. The response function matrix really needs three dimensions to handle the problem correctly. With larger computers than our VAX $11 / 780$, the method could be extended to handle multiple lines simultaneously. An obvious goal would be to try to obtain simultaneous images of active stars using photospheric, chromospheric, and transition region lines from the UV to the IR. Also, with large enough computers, large pieces of the spectrum could be imaged at once, including perhaps hundreds of lines. Including so many lines at once might help ease the present high $\mathrm{S} / \mathrm{N}$ per pixel requirements. There is the possibility that Doppler imaging could be done using linearly and/or circularly polarized light. Such 'Stokes parameter imagery' could provide information on magnetic fields in starspots, and on Ap stars.

Besides Ap stars, and spotted late-type stars, there are other potentially interesting classes of objects which may be amenable to the Doppler imaging technique. Imaging of accretion disks in cataclysmic variable systems has already been done using maximum entropy and broadband photometry. Several groups are at work using spectroscopy to Doppler image the disks. It should be possible to use the technique to derive accurate images of contact and nearcontact binary systems. With eclipsing binaries, the occulting star offers a further probe which could be incorporated into the imaging technique. Finally, the in- 
creased light gathering power of the next generation of large telescopes will bring many of the T Tauri and other pre-main sequence spotted stars within reach of the Doppler imaging technique, allowing us to obtain direct images of the some of the spectacular activity which occurs on such stars.

I would like to thank Dr. Keith Horne for bringing MEMSYS to our attention, and Dr. Robert Kraft and the Lick Time Allocation Committee for generous amounts of observing time in support of this effort. Much of the instrumentation which makes this work possible was funded by NSF block grants AST-8320396 and AST- 8614510. The Doppler imaging research effort at Lick is funded entirely by NSF grant AST-8210202 whose support we gratefully acknowledge.

\section{REFERENCES}

Deutsch, A.J. 1958, in IAU Symposium 6, Electromagnetic Phenomena in Cosmical Physics, ed. B. Lehnert (Cambridge: Cambridge University Press), p. 209.

Deutsch, A.J. 1970, Ap. J., 159, 985.

Falk, A.E., and Wehlau, W.H. 1974, Ap. J., 192, 409.

Goncharsky, A.V., Stepanov, V.V., Khokhlova, V.L., and Yagola, A.G. 1982, Astr. Zh., 59, 1146 (English transl. in Soviet Astr., 26, 690.)

Gull, S.F., and Skilling, J. 1983, in Indirect Imaging ed. J.A. Roberts (Cambridge: Cambridge University Press), p. 267.

Khokhlova, V.L. 1975, Astr. Zh., 52, 950 (English transl. in Soviet Astr., 19, 576.)

Khokhlova, V.L. 1985, Ap. Space Phys. Rev., 4, 99.

Khokhlova, V.L., and Ryabchikova, T.A. 1975, Astrophys. Space Sci., 34, 403.

Khokhlova, V.L., Rice, J.B., and Wehlau, W.H. 1986, Ap. J., 307, 768.

Kurucz, R.L. 1979, Ap. J. Suppl., 40, 1.

Megessier, C. 1975, Astr. Ap., 39, 263.

Pyper, D.M. 1969, Ap. J. Suppl., 18, 347.

Robinson, L.B., and Osborne, J. 1986, S.P.I.E., 627, 492.

Shore, J.E., and Johnson, R.W. 1980, IEEE Trans., IT-26, 26.

Shore, J.E., and Johnson, R.W. 1983, IEEE Trans., IT-29, 942.

Skilling, J., and Bryan, R.J. 1984, M.N.R.A.S., 211, 111.

Vaughan, A.H., Baliunas, S.L., Middlekoop, F., Hartmann, L., Mihalas, D., Noyes, R.W., and Preston, G.W. 1981, Ap. J., 250, 276.

Vogt, S.S. 1983, in IAU Colloquium 71, Activity in Red-Dwarf Stars, ed. M. Rodono and P. Byrne (Dordrecht: Reidel), p. 137.

Vogt, S.S., and Penrod, G.D. 1983a, in IAU Colloquium 71, Activity in Red-Dwarf Stars, ed. M. Rodono and P. Byrne (Dordrecht: Reidel), p. 379.

Vogt, S.S., and Penrod, G.D. 1983b, Pub. A.S.P., 95, 565.

Vogt, S.S., Penrod, G.D., and Hatzes, A. 1987, Ap.J., in press. 


\section{DISCUSSION}

FOING: In your constraint on the solution, you maximize $\mathrm{Q}=\mathrm{S}-\lambda \chi^{2}$. How and what is the choice of the Lagrange multiplier between the constraint of maximum entropy and minimal $\mathrm{X}^{2}$ distance to the data. How arbitrary is it for the stellar case?

VOGT: The entropy and $\chi^{2}$ terms can be weighted individually. We set up the method to give very high weight to the $\chi^{2}$ term, thus forcing the method to fit the data to very high accuracy (as is appropriate for high $\mathrm{S} / \mathrm{N}$ data).

FOING: Do you need simultaneous photometry and how do you correct for the secondary if it is variable, or if you do not know the changes of continuum level of the primary?

VOGT: We do not use simultaneous photometry. If the lines of the secondary were variable, the deblending would become impossible. The principal effect of changing stellar brightness will be a variable equivalent width of the lines, a quantity we do not really exploit in the imaging of spotted late type stars.

LINSKY: How secure is the indentification of the large polar spot on HR 1099?

VOGT: The polar spot is very well determined, if our input physics is correct. The presence of large polar spots on these stars is further supported by a correlation between line profile shapes and inclination. HD $199178\left(\mathrm{i}>60^{\circ}\right)$ does not show very pronounced polar spot effects. HD $26337\left(\mathrm{i} \sim 45^{\circ}\right)$ always has quite flat-bottomed profiles, and HR $1099\left(\mathrm{i}=33^{\circ}\right)$ also has very pronounced profile variations from the polar spot.

FURENLID: Do you feel that the polar spots are determined with the same confidence as the low-latitude spots, considering that there are no Doppler shifts for the polar spots?

VOGT: Yes, in fact they are generally better determined. They occupy the region of the star which is always in view, and thus has the largest amount of information in the line profiles. One does not need a Doppler shift to get information. The lack of Doppler shift contains the same information. Spots at very low latitudes have large shifts, but are not visible for as long, and thus (with finite phase coverage) are often less well determined.

SODERBLOM: To achieve high $\mathrm{S} / \mathrm{N}$ with a CCD, must one spread the spectrum over a number of pixels? What is the best $\mathrm{S} / \mathrm{N}$ per pixel achievable, in your experience?

VOGT: It certainly helps to spread the light out, but I have never done any tests to see how well one could do using only a few rows. One would quickly run into the dynamic range limit of the horizontal shift register. The highest $\mathrm{S} / \mathrm{N}$ we routinely achieve with the Texas Instruments $800 \times 800 \mathrm{CCD}$ is somewhere around 400-500. Pixel-to-pixel variations calibrate out much better than variations on scales of 10's to 100's of pixels. We have not tried to push $\mathrm{S} / \mathrm{N}$ further, but I would not be surprised that, with some care, much higher S/N's could be achieved with this CCD. 